
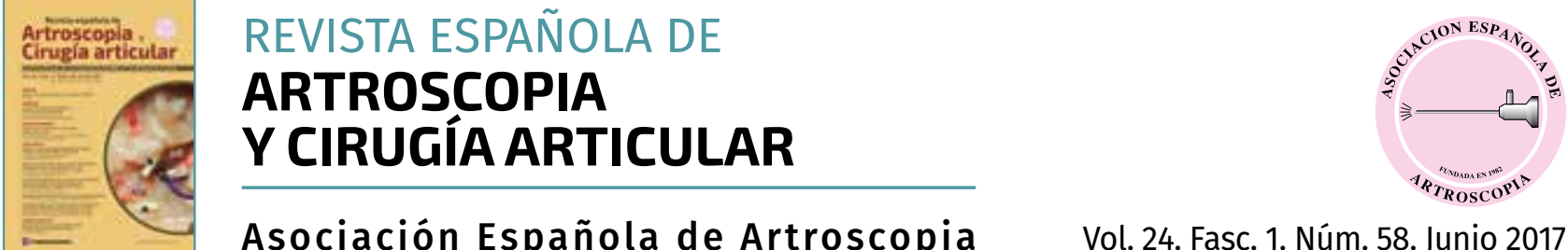

Vol. 24. Fasc. 1. Núm. 58. Junio 2017

ISSN: 2386-3129 (impreso)

2443-9754 (online)

\title{
Original
}

\section{Agenesia bilateral del tendón de la porción larga del bíceps}

\author{
A. Ron Marqués', F. Canillas del Rey², P. Menéndez Martínez² \\ Traumatología Vistahermosa. Clínica Vistahermosa. Alicante \\ Correspondencia: \\ Dr. Fernando Canillas del Rey \\ Correo electrónico: fercanillas@yahoo.es
}

Recibido el 3 de abril de 2017

Aceptado el 6 de abril de 2017

Disponible en Internet: junio 2017

\section{RESUMEN}

Presentamos el caso de un paciente varón de 49 años intervenido por presentar un síndrome subacromial derecho crónico refractario a tratamiento médico y rehabilitador. En el tiempo artroscópico intraarticular se apreció la ausencia completa del tendón de la porción larga del músculo bíceps braquial, que no se había objetivado en el estudio clínico y de imagen previo a la cirugía. En el postoperatorio se estudió el hombro contralateral y se comprobó la ausencia bilateral de la porción larga del músculo bíceps braquial. Dada la infrecuencia de esta anomalía, se revisa la bibliografía y su patogenia.

Palabras clave: Hombro. Porción larga músculo bíceps braquial. Artroscopia. Agenesia. Sindrome subacromial.

\section{Introducción}

El tendón de la porción larga del músculo bíceps braquial (TPLB) es causa de dolor de hombro debido a patologías de tipo degenerativo o traumático, como tendinosis, tendinitis, subluxaciones y roturas parciales o completas. La agenesia del TPLB es una patología infrecuente que se puede presentar de forma uni- o bilateral, pudiéndose asociar otras anomalías congénitas. La exploración clínica exhaustiva, las pruebas de imagen y

\begin{abstract}
Bilateral absence of the long head of the biceps tendon

A 49-years-old male patient presented with chronic right subacromial syndrome refractory to conventional medical and rehabilitation treatment, who undenwent arthroscopic surgery. Unexpectedly no long head of the muscle biceps brachii tendon was found during the arthroscopic intraarticular evaluation, even though it's absence was not reported in the previous clinical examination or radiological tests. This infrequent anomaly leads us to review the literature and it's pathogenesis.
\end{abstract}

Key words: Shoulder. Long head of the muscle biceps brachii. Arthroscopy. Agenesis. Subacromial syndrome.

su confirmación artroscópica son los pilares fundamentales para el diagnóstico. Presentamos un caso de agenesia bilateral bicipital en paciente con omalgia crónica y revisamos la bibliografía.

\section{Caso clínico}

Se trata de un varón de 49 años de edad que consultó por hombro izquierdo doloroso crónico. No refirió traumatismo o esfuerzos. Como único an-

https://doi.org/10.24129/j.reaca.24158.fs1704016

FS $\odot 2017$ Fundación Española de Artroscopia. Publicado por Imaidea Interactiva en FONDOSCIENCE ${ }^{\circledR}$ (www.fondoscience.com). Este es un artículo Open Access bajo la licencia CC BY-NC-ND (www.creativecommons.org/licenses/by-nc-nd/4.0/). 


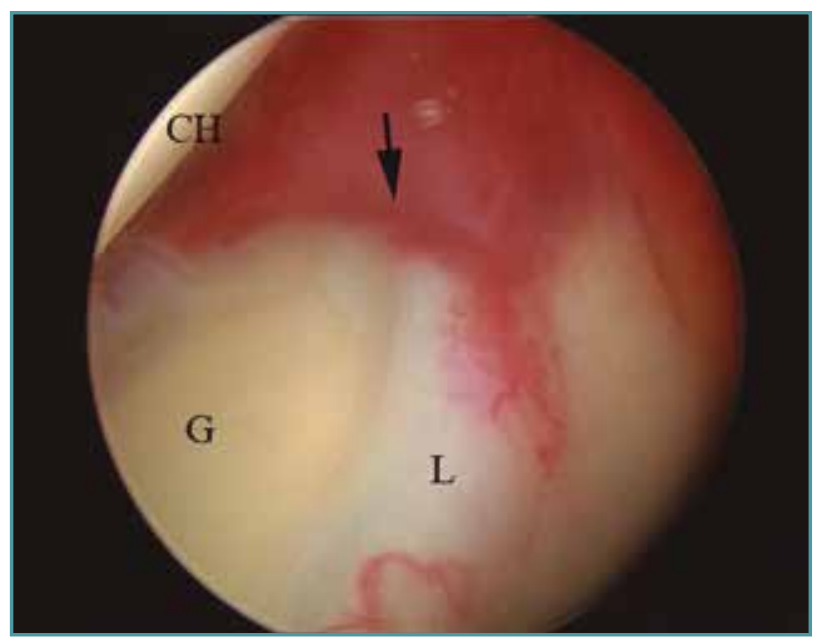

Figura 1. Imagen intraarticular desde el portal posterior del hombro izquierdo (en decúbito lateral). Se objetiva la ausencia de porción larga del tendón del músculo bíceps braquial (flecha). CH: cabeza humeral; G: glena; L: labrum glenoideo.

tecedente presentaba una sordera congénita. El dolor era mecánico y aumentaba con movimientos cotidianos que le despertaban por la noche. No tenía sintomas en el otro hombro. Tomó antiinflamatorios y calmantes y realizó 4 ciclos de rehabilitación sin percibir mejoría. Fue infiltrado en 3 ocasiones con alivio del dolor de poca duración. En la exploración, no se objetivaron hipotrofias musculares. La movilidad activa era de $85^{\circ}$ de abducción, $90^{\circ}$ de antepulsión, rotación externa de $30^{\circ}$ e interna de $30^{\circ}$. La movilidad pasiva era completa pero dolorosa a partir de los grados antes señalados. Las maniobras exploratorias para afectación del tendón del músculo supraespinoso eran positivas, siendo negativas para el tendón del músculo bíceps braquial, del músculo infraespinoso y del músculo subescapular. No presentaba dolor en la articulación acromioclavicular ni tampoco había signos de inestabilidad glenohumeral. En la radiografía y en la resonancia magnética (RM) se apreció un acromion de tipo III de Bigliani y presencia de signos de tendinosis en el tendón del músculo supraespinoso. En el resto de las estructuras no se observaron alteraciones significativas.

Dada la mala respuesta al tratamiento médico, se decidió realizar una descompresión subacromial artroscópica. En la exploración intraarticular se objetivó la ausencia del TPLB (Figura 1) con un labrum sin muestra de rotura actual o antigua. Se realizó una bursectomía artroscópica con vaporizador y acromioplastia con motor artroscópico. El postoperatorio trascurrió sin incidencias, presentando una recuperación clínica y funcional completa. Ante el hallazgo casual de la agenesia del músculo bíceps braquial, se revisaron las imágenes preoperatorias de RM, confirmándose la ausencia del tendón en la corredera bicipital, la cual se observó hipoplásica (Figura 2). Se realizó un estudio ecográfico contralateral donde también se objetivó que la agenesia bicipital era bilateral y una corredera hipoplásica (Figura 2).
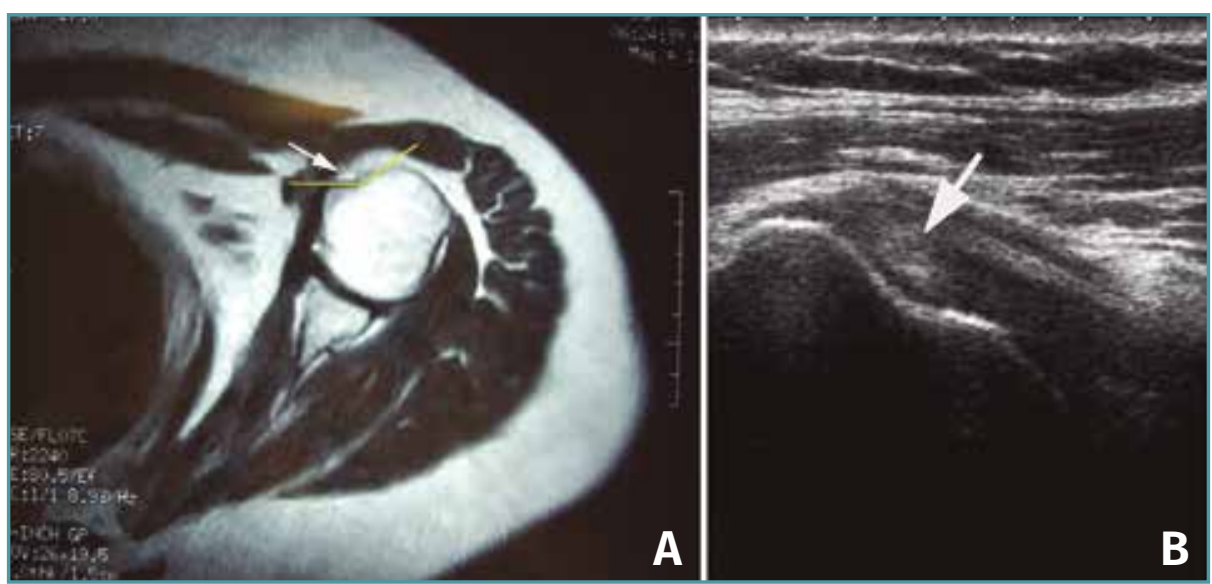

Figura 2. A: resonancia magnética $(R M)$ del hombro afecto. Se aprecia aumento del ángulo de la corredera bicipital y ausencia del tendón de la porción larga del bíceps; B: ecografía del hombro derecho (contralateral). Se observa ausencia de la porción larga del músculo bíceps braquial e hipoplasia de la corredera bicipital (flecha).

\section{Discusión}

El tendón del músculo biceps braquial es uno de los tendones con mayor variabilidad de la anatomía humana; el hallazgo más frecuente son las terminaciones supernumerarias y las variaciones en su origen intraarticular. La ausencia del TPLB es una anomalía infrecuente ${ }^{(1-5)}$. Revisando la bibliografía (Tabla 1) se encontraron 17 casos de agenesia, 6 


\begin{tabular}{|c|c|c|c|c|c|c|}
\hline & Sexo & Edad & Lado & Lesiones congénitas & Inestabilidad & Diagnóstico RM \\
\hline Mariani (1997) & Varón & 23 & Bilateral & No & - & No \\
\hline McDonald (1998) & Varón & 25 & Izquierdo & No & No & - \\
\hline Smith (2002) & Varón & 16 & Derecho & Sí (VATER) & $\begin{array}{l}\text { Sí } \\
\text { Hipoplasia de labrum y } \\
\text { glenohumeral medio e } \\
\text { inferior }\end{array}$ & No \\
\hline Glueck (2003) & Mujer & 25 & Izquierdo & No & $\begin{array}{c}\text { Sí } \\
\text { Multidireccional }\end{array}$ & No \\
\hline Franco (2005) & Varón & 37 & Derecho & $\begin{array}{c}\text { Sí } \\
\text { (espina bífida oculta, } \\
\text { hernia inguinal } \\
\text { congénita, testículo no } \\
\text { descendido }\end{array}$ & $\begin{array}{c}\text { Sí } \\
\text { Bankart + cápsula laxa }\end{array}$ & No \\
\hline Pritsch (2005) & Varón & 21 & Izquierdo & No & $\begin{array}{c}\text { Sí } \\
\text { Bankart + Hill-Sachs }\end{array}$ & No \\
\hline \multirow{3}{*}{ Ghalayini (2007) } & Mujer & 37 & Bilateral & Sí & $\begin{array}{c}\text { Sí } \\
\text { Bankart inferior + } \\
\text { glenohumeral inferior }\end{array}$ & Sí \\
\hline & Mujer & 28 & - & No & $\begin{array}{c}\text { Sí } \\
\text { SLAP }\end{array}$ & No \\
\hline & Mujer & 34 & - & No & $\begin{array}{c}\text { Sí } \\
\text { Rotura supraespinoso }\end{array}$ & Sí \\
\hline Keefe (2003) & Varón & 17 & Izquierdo & No & $\begin{array}{c}\text { Sí } \\
\text { SLAP (reparación) }\end{array}$ & Sí \\
\hline Sayeed (2008) & Varón & 18 & Derecho & $\begin{array}{c}\text { No } \\
\text { (displasia de glena } \\
\text { congénita) }\end{array}$ & $\begin{array}{c}\text { Sí } \\
\text { Rotura labrum posterior } \\
\text { (luxaciones voluntarias } \\
\text { en infancia) }\end{array}$ & Sí \\
\hline Koplas (2009) & Varón & 49 & Bilateral & No & $\begin{array}{c}\text { Sí } \\
\text { Supraespinoso y labrum } \\
\text { superior }\end{array}$ & No \\
\hline Kuhn (2009) & Varón & 30 & Bilateral & No & No & No \\
\hline Maldjian (2013) & Mujer & 42 & Bilateral & $\begin{array}{c}\text { Sí } \\
\text { (aplasia de radio) }\end{array}$ & $\begin{array}{c}\text { No } \\
\text { Bankart como hallazgo }\end{array}$ & Sí \\
\hline Gillardin (2013) & Varón & 22 & Derecho & Sí (VATER) & No & Sí \\
\hline Foad (2016) & Mujer & 24 & Derecho & No & $\begin{array}{c}\text { Sí } \\
\text { Labrum anteroinferior }\end{array}$ & No \\
\hline Rego (2015) & Varón & 29 & Bilateral & No & No & Sí \\
\hline Ron (2017) & Varón & 40 & Bilateral & No & No & Sí \\
\hline
\end{tabular}

de ellos bilaterales. Cinco de los pacientes asociaban alteraciones congénitas; de estos, solo 1 presentaba agenesia bilateral siendo, además, el único paciente con alteración aislada musculoesquelética (aplasia de radio)(4). Todos estos pacientes asociaban además inestabilidad; las alteraciones se produjeron en torno a la séptima semana de gestación, compartiendo anomalías del desarrollo capsular, tendón subescapular y labrum glenoideo debido al origen común de es- 
tas estructuras, lo que contribuiría a aumentar la inestabilidad glenohumeral.

Es necesario encontrar herramientas clínicas y pruebas imagen que nos ayuden en el diagnóstico diferencial entre lesión o agenesia del TPLB. Los test de O'Brien y Speed no son buenos para diagnosticar lesión aislada del tendón bicipital y suelen ser positivos cuando se añaden alteraciones del manguito. La ausencia del signo de Popeye y la falta de dolor a la palpación de la corredera bicipital son signos constantes en todos los estudios $^{(1,2)}$. En la ecografía y la RM, los signos claves son falta de retracción del vientre muscular del bíceps, hipoplasia del surco bicipital ${ }^{(1,2)}$ e hipertrofia del ligamento semicircular del húmero(4). En nuestro caso, se trató de un hallazgo artroscópico casual, sin ser objetivado en la RM preoperatoria, que se confirmó posteriormente ante la del TPLB y una corredera bicipital hipoplásica. El estudio ecográfico del hombro contralateral confirmó que se trataba de una agenesia bilateral.

Existe controversia sobre la importancia de la agenesia de la PLB en la estabilidad del hombro. Estudios de electromiografía y en cadáver demuestran la función del TPLB como estabilizador dinámico en la traslación anterior, disminuyendo las solicitaciones sobre el ligamento glenohumeral inferior y siendo un depresor fundamental de la cabeza humeral en los movimientos de abducción y rotación externa ${ }^{(1,3,5)}$. A pesar de que hay estudios en los que la agenesia de la PLB no está asociada a inestabilidad u otras alteraciones congénitas ${ }^{(3)}$, la mayoría describen cómo su ausencia es un factor que contribuye a la microinestabilidad por fracaso en sus funciones, que si se mantiene provocará clínica. En contraposición a esta hipótesis de la microinestabilidad, en todos los pacientes que fueron tratados por dolor e inestabilidad en los que la agenesia de biceps fue un hallazgo artroscópico, el tratamiento de las lesiones que asociaban y generaban dicha inestabilidad eliminó el dolor, sin añadirse gestos sobre la PLB $B^{(1,2,4)}$.

El caso que presentamos coincide con otros en que el diagnóstico se realizó durante el tiempo quirúrgico y no en la exploración clínica o en los estudios previos con RM. No presentaba signos de inestabilidad y el resultado de la descompresión artroscópica nos sugiere que el papel de la agenesia es controvertido en la patogénesis del dolor crónico del hombro y de la inestabilidad.

La agenesia de PLB es una patología infrecuente que se presenta de forma uni- o bilateral, asociada o no a alteraciones congénitas. Es un reto diagnóstico, siendo muchas veces hallazgo casual en pacientes con omalgia, con o sin inestabilidad, a los que se indica tratamiento artroscópico. Algunos indicios nos pueden ayudar en el diagnóstico diferencial, como la ausencia del signo de Popeye, la falta de retracción del vientre muscular del bíceps o la hipoplasia de la corredera bicipital.

Aunque está claramente definida la función del TPLB en la estabilidad del hombro, existe controversia en las publicaciones sobre la asociación entre agenesia e inestabilidad. En pacientes, como el caso que presentamos, con dolor y sin inestabilidad, se plantea que la agenesia genere por sí misma una microinestabilidad que justifique los sintomas sin asociar otras lesiones.

\section{Bibliografía}

1. Foad A, Faruqui S. Case Report: Absence of the Long Head of the Biceps Brachii Tendon. Iowa Orthop J. 2016;36:88-93.

2. Rego Costa F, Esteves C, Melão L. Bilateral Congenital Agenesis of the Long Head of the Biceps Tendon: The Beginning. Case Rep Radiol. 2016;2016:4309213.

3. Wittstein J, Lassiter T Jr, Taylor DJ. Aberrant origin of the long head of the biceps: a case series. Shoulder Elbow Surg. 2012;21:356-60.

4. Maldjian C, Borrero C, Adam R, Vyas D. Nature abhors a vacuum: bilateral prominent rotator cable in bilateral congenital absence of the long head of the biceps tendon. Skelet Radiol. 2014;43:75-8.

5. Ghalayini SR, Board TN, Srinivasan MS. Anatomic variations in the long head of biceps: contribution to shoulder dysfunction. Arthroscopy. 2007;23:1012-8. 